\title{
Determination of amino acid profile for argininosuccinic aciduria disorder using High-Performance Liquid Chromatography with fluorescence detection
}

\author{
Hossein Salmanizadeh ${ }^{\otimes}$ and Neda Sahi² \\ Mahdieh Medical Diagnostic Centre, Isfahan, Iran; 2Department of Plant and Animal Biology, Faculty of Biological Science and Technology, \\ University of Isfahan, Isfahan, Iran
}

Argininosuccinic aciduria is an autosomal, recessive amino acid disorder that is caused by a deficiency of the argininosuccinate lyase enzyme. Citrulline is the most significant marker to detect this disorder. We used the High-performance liquid chromatography with fluorescence detection with $450 \mathrm{~nm}$ emission and $330 \mathrm{~nm}$ excitation wavelengths, $15 \mathrm{mmol} / \mathrm{L}$ potassium dihydrogen phosphate and $5 \mathrm{mmol} / \mathrm{L}$ dipotassium hydrogen phosphate as Mobile Phase A, and $50 \mathrm{~mL}$ water, $250 \mathrm{~mL}$ acetonitrile, and $200 \mathrm{~mL}$ methanol as Mobile Phase B in gradient mode with flow rate of $1.2 \mathrm{~mL} / \mathrm{min}$. The citrulline concentration was $22 \mu \mathrm{mol} / \mathrm{L}$ in healthy infants and 220 $\mu \mathrm{mol} / \mathrm{L}$ in infants suffering from the disorder.

Key words: argininosuccinic aciduria, citrulline, amino acid profile

Received: 15 January, 2020; revised: 27 August, 2020; accepted: 30 August, 2020; available on-line: 14 September, 2020

๑e-mail: Salmanizadeh64@yahoo.com

Acknowledgements of Financial Support: This research project was funded by Mahdieh Medical Diagnostic Centre, Isfahan, Iran.

Abbreviations: ASL, argininosuccinate lyase enzyme; HPLC-FD, High-Performance Liquid Chromatography-Fluorescence detector method; DBS, Dried Blood Spot; HPLC-PDA, High-Performance Liquid Chromatography-Photodiode Array Detector method; RP-HPLC, Reverse-Phase High-Performance Liquid Chromatography method; HPLC-UV, High-Performance Liquid Chromatography-Ultra-violet/ Visible Detector method; OPA, O-phthalaldehyde solution; MPA, 3-mercaptopropionic acid solution; EDTA, ethylenediaminetetraacetic acid

\section{INTRODUCTION}

There are more than three-hundred amino acids in the natural environment. They can be classified into several categories such as $\alpha$-amino acids, $\beta$-amino acids, $\gamma$-amino acids, $\delta$-amino acids, etc. based on the position of the amino group bonded to the carboxyl group in the amino acid structure (Nelson et al., 2017; Sharer et al., 2018). $\mathrm{L}-\alpha$-amino acids can be combined by peptide bonds to produce proteins in the human body (Nelson et al., 2017; Sharer et al., 2018).

Common amino acids (twenty amino acids) can be divided into two categories: essential amino acids (nine amino acids: histidine, leucine, isoleucine, lysine, methionine, phenylalanine, threonine, tryptophan, and valine) and non-essential amino acids (ten amino acids: alanine, aspartate, asparagine, glutamate, glutamine, serine, tyrosine, proline, glycine, and arginine) (Nelson et al., 2017; Sharer et al., 2018). The essential amino acids, in contrast to non-essential amino acids, are not produced de novo in humans and all animals and have to be taken with the diet (Sharer et al., 2018). Also, common amino acids and their derivatives play significant roles in processes such as the production of several hormones, biosynthesis of porphyrins, nucleotides (purines and pyrimidines), polyamines, sulfur compounds, and neurotransmitters (Nelson et al., 2017). Because of the different roles of amino acids, every disturbance of amino acid metabolism results in serious health disorders (Nelson et al., 2017).

Congenital or hereditary metabolic disorders are a group of diseases, which includes amino acid metabolism disorders. The disorders used to be considered as rare diseases, but nowadays they are well-known (Martin et al., 2006). Based on the severity and intensity, amino acid disorders can be classified into several types, each of them resulting in long-lasting physiological damage of infants' and children's bodies (Martin et al., 2006). The urea cycle is one of the most important pathways of amino acid metabolism and takes place in the liver; it leads to ammonia (produced in amino acid catabolism) conversion into urea. The cycle includes six enzymatic reactions, and any disturbance of this pathway leads to amino acid metabolism disorder (Erez, 2013; Kim et al., 2018; Martin et al., 2006; U.S. National Library of Medicine).

Argininosuccinate lyase (ASL) enzyme is the third enzyme acting in the urea cycle. ASL enzyme converts argininosuccinic acid into arginine and fumarate, so any deficiency of this enzyme results in argininosuccinic aciduria disorder (an autosomal recessive disease) (Fig. 1) (Erez, 2013; Kim et al., 2018). The disease occurs in ap-

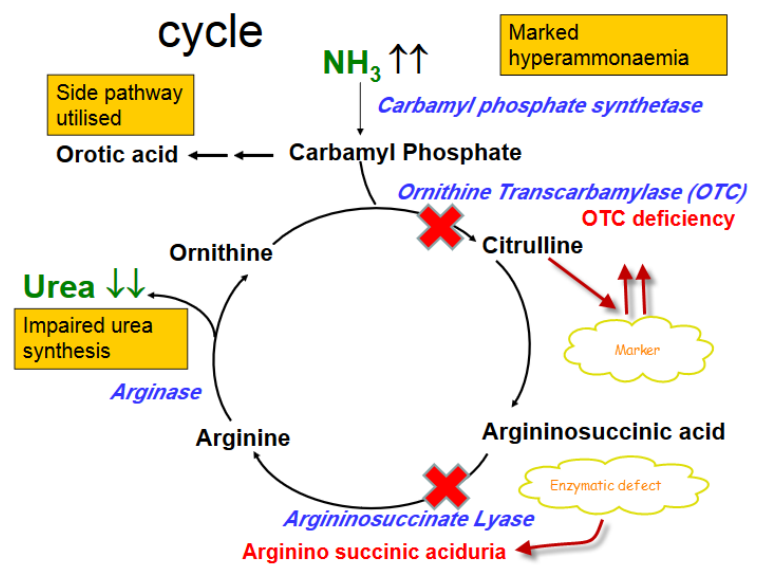

Figure 1. The urea cycle and argininosuccinic aciduria disorder. The activity of argininosuccinic lyase is diminished in this pathway. It results in increased citrulline level which is the best marker to determine by HPLC-FD. 
proximately one in 70000 to 218000 newborns around the world (U.S. National Library of Medicine). The first case of this disease was reported in 1958 and involved severe mental deficiency (Levin et al., 1961). There are two phenotypic forms of argininosuccinic aciduria: neonatal acute form (the classical form) and milder late-onset form (Biase et al., 2015; Chin Chen, 2010). In the first form, infants usually seem normal and healthy during the first day after birth, but show several symptoms such as poor nutrition, lethargy, tiredness and irregular breathing due to ammonia accumulation (Hyperammonemia) over the next few days. Also, without treatment, they fall into a coma and die (Chin Chen, 2010; Nagamani et al., 2012). The second form occurs following an acute viral infection or stress in children; therefore, it can be diagnosed not only at birth but also at any age. In addition, infants show several symptoms such as anorexia, vomiting, developmental disabilities, seizures, and behavioral problems (Chin Chen, 2010; Nagamani et al., 2012).

There are several markers to identify these two types of argininosuccinic aciduria. The increased level of citrulline is the most crucial marker, and it is detected using the amino acid profile. Hyperammonemia (especially in the first form of the disease) is another important marker, and it is also detected by the amino acid profile. Nowadays, there are some techniques to acquire and analyze the amino acid profile, and the High-performance liquid chromatography with fluorescence detection (HPLC-FD) technique is one of the most reliable methods to do this.

Chin Chen and others (Chin Chen et al., 2010) studied argininosuccinic aciduria disorder using ion-exchange chromatography with a dedicated amino acid analyzer (Biochrom 30) and post-column detection. Farideh Haghighi and others (Haghighi et al., 2015) determined phenylalanine and tyrosine concentration from Dried Blood Spot (DBS) using a Gradient High-Performance Liquid Chromatography with Photodiode Array Detection (HPLC-PDA). Tcherkas \& Denisenko (Tcherkas \& Denisenko, 2001) reported a method to measure homocysteine and cysteine as well as aspartate and glutamate level by Reverse-Phase High-Performance Liquid Chromatography (RP-HPLC). Roman Kand'ar \& Pavla Zakova (Roman Kand'ar \& Pavla Zakova, 2009) measured phenylalanine and tyrosine concentration in plasma and DBS samples using HPLC-FD. Ying Li and others (Ying Li et al., 2011) measured aromatic amino acids by HPLC-FD. Also, Xi-ming Mo and others (Mo et al., 2013) determined phenylalanine and tyrosine levels in peripheral capillary blood using High-Performance Liq-

Table 1. Gradient Mode for HPLC method

\begin{tabular}{llll}
\hline Time $(\mathrm{min})$ & $\mathrm{A}(\%)$ & $\mathrm{B}(\%)$ & Flow rate $(\mathrm{mL} / \mathrm{min})$ \\
\hline Initial & 100 & 0 & 1.2 \\
\hline 5 & 74 & 26 & 32 \\
\hline 10 & 68 & 36 & 1.2 \\
\hline 13 & 64 & 40 & 1.2 \\
\hline 16 & 60 & 50 & 1.2 \\
\hline 22 & 50 & 55 & 1.2 \\
\hline 25 & 45 & 75 & 100 \\
\hline 28 & 25 & 0 & 1.2 \\
\hline 29 & 100 & 1.2 \\
\hline 30 & 0 & 1.2 \\
\hline
\end{tabular}

uid Chromatography with Ultra-violet/Visible Detection (HPLC-UV).

\section{MATERIALS AND METHODS}

Chemical materials. Ultrapure water for chromatography (2.5 L, Merck, $\mathrm{H}_{2} \mathrm{O}$, MW: $18.02 \mathrm{~g} / \mathrm{mol}$ ), Boric acid ( $1 \mathrm{~kg}$, Merck, $\left.\mathrm{H}_{3} \mathrm{BO}_{4}, \mathrm{MW}: 61.83 \mathrm{~g} / \mathrm{mol}\right)$, sodium hydroxide pellets $(1 \mathrm{~kg}$, Merck, $\mathrm{NaOH}, \mathrm{MW}: 40 \mathrm{~g} / \mathrm{mol})$, 3-mercaptopropionic acid $\left(100 \mathrm{~mL}\right.$, Merck, $\mathrm{C}_{3} \mathrm{H}_{6} \mathrm{O}_{2} \mathrm{~S}$, MW: $106.14 \mathrm{~g} / \mathrm{mol}, 98 \%$ ), O-phthalaldehyde (5 g, Merck, $\left.\mathrm{C}_{6} \mathrm{H}_{4}(\mathrm{CHO})_{2} \mathrm{MW}: 134.134 \mathrm{~g} / \mathrm{mol}\right)$, 9-fluorenylmethyl chloroformate $\left(5 \mathrm{~g}\right.$, Merck, $\mathrm{C}_{15} \mathrm{H}_{11} \mathrm{CLO}_{2}, \mathrm{MW}: 258.7 \mathrm{~g} /$ mol), acetonitrile (2.5 L, Merck, $\mathrm{CH}_{3} \mathrm{CN}, \mathrm{MW}: 41.05$ $\mathrm{g} / \mathrm{mol}$ ), phosphoric acid (2.5 L, Merck, $\mathrm{H}_{3} \mathrm{PO}_{4}, 85 \%$, MW: $97.995 \mathrm{~g} / \mathrm{mol})$, potassium dihydrogenphosphate (1 kg, Merck, $\left.\mathrm{KH}_{2} \mathrm{PO}_{4}, \mathrm{MW}: 136.089 \mathrm{~g} / \mathrm{mol}\right)$, dipotassium hydrogen phosphate $\left(1 \mathrm{~kg}\right.$, Merck, $\mathrm{K}_{2} \mathrm{HPO}_{4}, \mathrm{MW}$ : $174.18 \mathrm{~g} / \mathrm{mol})$, ortho-phosphoric acid (2.5 L, Merck, $\left.\mathrm{H}_{3} \mathrm{PO}_{3}, 85 \%, \mathrm{MW}: 98.00 \mathrm{~g} / \mathrm{mol}\right)$, ethanol (2.5 L, Merck, $\mathrm{C}_{2} \mathrm{H}_{3} \mathrm{OH}, \mathrm{MW}: 46.07 \mathrm{~g} / \mathrm{mol}, 99.9 \%$,), methanol $(2.5 \mathrm{~L}$, Merck, $\mathrm{CH}_{3} \mathrm{OH}, \mathrm{MW}: 32.04 \mathrm{~g} / \mathrm{mol}, 99.9 \%$ ), amino acid analytical standard (AA18, $1 \mathrm{~mL}$, Sigma Aldrich), L-citrulline powder $\left(5,25 \mathrm{~g}\right.$, Merck, $\mathrm{C}_{6} \mathrm{H}_{13} \mathrm{~N}_{3} \mathrm{O}_{3}, \mathrm{MW}: 175.19$ $\mathrm{CO}_{2} \mathrm{H}$, , MW: $\left.131.17 \mathrm{~g} / \mathrm{mol}\right)$ were purchased.

System configuration. Solvent delivery: pump $6.1 \mathrm{~L}$, Azura Knauer (Germany). Fluorescence detector: Prominence, RF-20A, Shimadzu Corp. (Japan). Column oven: CTO-20A, Prominence, Shimadzu Corp. (Japan). Injection valve: Rheodyne7725i, Perkin Elmer (USA) fitted with a $50 \mu \mathrm{L}$ sample-loading loop. Amino acid column: 150/4.6, Nucleodur C18, Gravity $5 \mu \mathrm{m}$, Macherey-Nagel (Germany). LC workstation: Clarity software version 5. Syringe: SGE $50 \mu \mathrm{l}$ (Australia).

Mobile phases. Borate buffer $0.1 \mathrm{~mol} / \mathrm{L}: 3.1 \mathrm{~g}$ of boric acid and $1 \mathrm{~g}$ of sodium hydroxide dissolved in 500 $\mathrm{mL}$ ultrapure water. OPA reagent (O-phthalaldehyde): $20 \mathrm{mg}$ of O-phthalaldehyde dissolved in $1 \mathrm{~mL}$ of methanol, and mixed well by sonication; then, $2 \mathrm{~mL}$ of borate buffer and $12 \mathrm{~mL}$ of ultrapure water was added. MPA reagent (3-mercaptopropionic acid solution): $20 \mu \mathrm{L}$ of 3 -mercaptopropionic acid dissolved in $20 \mathrm{~mL}$ of borate buffer. Mobile Phase A: $15 \mathrm{mmol} / \mathrm{L}$ potassium dihydrogen phosphate and $5 \mathrm{mmol} / \mathrm{L}$ dipotassium hydrogen phosphate. First, $1.02 \mathrm{~g}$ potassium dihydrogen phosphate was added to $0.43 \mathrm{~g}$ dipotassium hydrogen phosphate; next, mixed with $500 \mathrm{~mL}$ ultrapure water. Mobile Phase B: $50 \mathrm{~mL}$ ultrapure water, $250 \mathrm{~mL}$ acetonitrile and 200 $\mathrm{mL}$ methanol (v/v) mixed. Gradient mode with flow rate of $1.2 \mathrm{~mL} / \mathrm{min}$ was used (Table 1$)$. $\mathrm{g} / \mathrm{mol})$, L-norleucine (100mg, Merck, $\mathrm{CH}_{3}\left(\mathrm{CH}_{2}\right)_{3} \mathrm{CH}\left(\mathrm{NH}_{2}\right)$ 


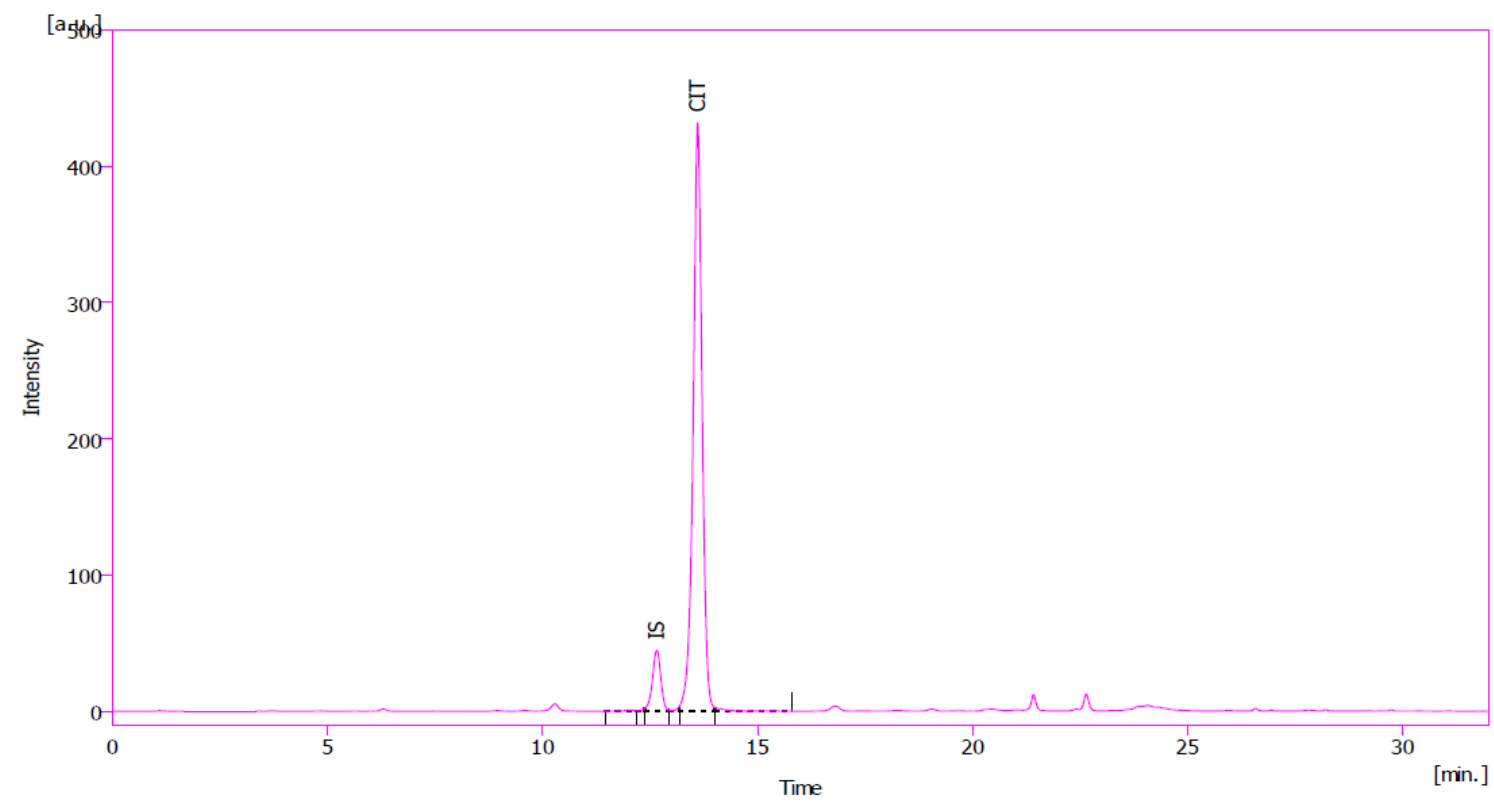

Figure 2. Citrulline standard.

The acquisition of this curve was performed using our protocol.

Citrulline standard. $0.0218 \mathrm{~g}$ of citrulline powder was dissolved in $50 \mathrm{~mL}$ ultrapure water $(0.1 \mathrm{~N} \mathrm{HCl})$. $50 \mu \mathrm{L}$ L-norleucine was added to $200 \mu \mathrm{L}$ of this standard solution as an internal standard. Next, $800 \mu \mathrm{L}$ of methanol was added, and the solution was centrifuged at $4000 \mathrm{rpm} .200 \mu \mathrm{L}$ standard, $100 \mu \mathrm{L}$ MPA reagent, and $50 \mu \mathrm{L}$ OPA reagent were mixed together. After $2 \mathrm{~min}$, $50 \mu \mathrm{L}$ of this sample was injected through Rheodyne injector into the HPLC unit. A single peak for citrulline standard was detected by HPLC-FD (Fig. 2).

Human plasma. First, blood for amino acid profile testing was collected into an anticoagulant ethylenediaminetetraacetic acid (EDTA) tube. Then, the tubes were centrifuged at $4000 \mathrm{rpm}$ and plasma was separated from other blood components for amino acid testing.

Sample pretreatment. First, we added $50 \mu \mathrm{l}$ of Lnorleucine to $200 \mu \mathrm{l}$ of human plasma as an internal standard. Then, $800 \mu \mathrm{l}$ of methanol was added and the mixture was centrifuged at $4000 \mathrm{rpm}$ to remove the precipitate. To determine the amino acid profile with HPLC reverse phase C18 column we used the liquid supernatant.

Precolumn derivatization. $200 \mu \mathrm{l}$ of the supernatant from the sample pretreatment step was mixed with 100 $\mu \mathrm{l}$ of MPA reagent, and $50 \mu \mathrm{l}$ of OPA. After $2 \mathrm{~min}, 50$ $\mu l$ of the final sample was injected through the Rheodyne injector into the HPLC unit.

Fluorescence detectable compounds. There are two main reactions to produce a fluorescent product. First, the OPA solution reacts with the amino groups in the amino acid structure, and generate the intermediate product. Second, the MPA solution reacts with the intermediate product, and it results in producing a fluorescent derivative. Finally, the fluorescent derivative is detected by the fluorescence detector and the amino acid concentration is determined (Fig. 3).

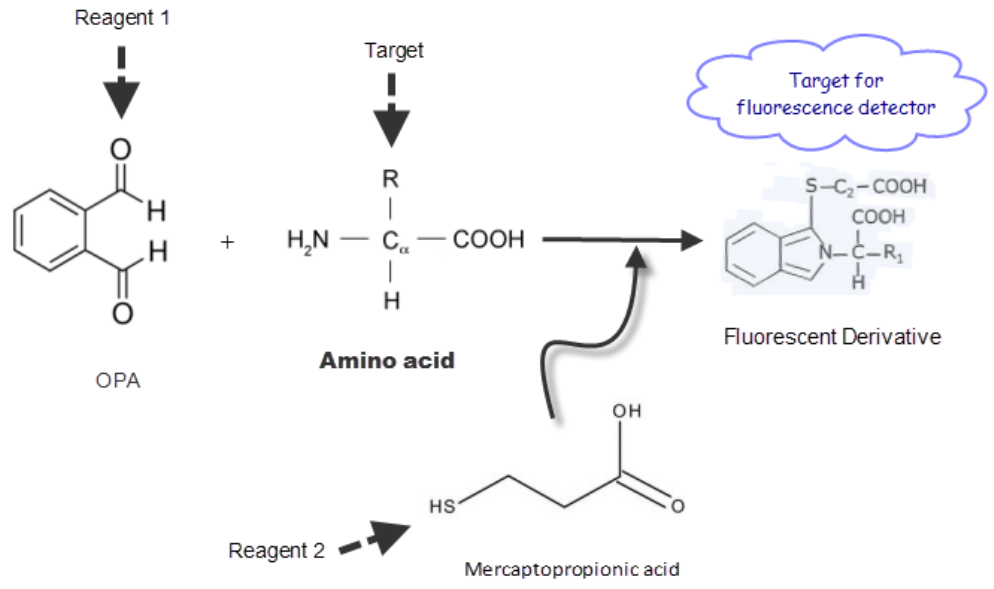

Figure 3. Precolumn derivatization analysis of amino acids with HPLC.

Amino acid reaction: amino acids are converted to the fluorescent derivative with O-phthalaldehyde (OPA) and 3-mercaptopropionic acid (MPA) reagents. 


\section{RESULTS AND DISCUSSION}

Congenital amino acid metabolic disorders are a group of metabolic disorders that have been diagnosed more rapidly in recent years due to advances in technology and the higher awareness of physicians. Previously, biochemical methods were used to identify these disorders (Sharer et al., 2018). However, they have some disadvantages such as being qualitative and useful only in diagnosis for common disorders (such as PKU). Nowadays, some accurate methods have become available to detect amino acid disorders. One of the most accurate methods is HPLC-FD. Argininosuccinic aciduria or argininosuccinic acidemia is the second most common urea cycle disorder, characterized by insufficient ASL enzyme activity. The ASL gene is located on chromosome 7 (7q.11.2) (Chin Chen, 2010) and its mutation results in this disease (autosomal recessive disease). There are two phenotypic forms of the disease: neonatal acute form (classical form) that occurs within the 24 hours from birth and milder late-onset form that develops later (Chin Chen, 2010; Biase et al., 2015; Nagamani et al., 2012). The amino acid profile of a six-year-old boy with the milder lateonset form of argininosuccinic aciduria was presented in Fig. 4.

The first and the most significant factor detected by the method we used was high citrulline level (220 $\mu \mathrm{mol} / \mathrm{L})$, comparing to $150-250 \mu \mathrm{mol} / \mathrm{L}$ in Chin Chen research (Chin Chen, 2010) and $100-300 \mu \mathrm{mol} / \mathrm{L}$ in Feuchtbaum and others work (Feuchtbaum et al., 2012). Another amino acid marker elevated in the disease was glutamine, reaching $850 \mu \mathrm{mol} / \mathrm{L}$. Glutamine is crucial as it eliminates ammonia from the bloodstream in the human body. Another non-specific amino acid that was slightly elevated was alanine. Recently, other symptoms of the disease were also noted such as some electrolyte imbalances, hypokalemia especially in infants not subjected to an appropriate therapy (Biase et al., 2015; Nagamani et al., 2012; Feuchtbaum et al., 2012). As hair naturally contains $10.5 \%$ arginine, its deficiency can also cause hair fragility in patients (Feuchtbaum et al., 2012). In addition, liver problems may also occur: from hepatomegaly to elevated liver enzymes level (Nagamani et al., 2012). Furthermore, in some patients, hypertension was recently observed (Nagamani et al., 2012). There are three main therapeutic approaches: (1) dietary restrictions to lower the protein intake, (2) administration of ammonia-eliminating drugs such as sodium benzoate, sodium phenylbutyrate and (3) oral supplementation of arginine (U.S. National Library of Medicine).

Farideh Haghighi and others (Haghighi et al., 2015) performed the HPLC-PDA to detect two amino acids, namely phenylalanine and tyrosine, in DBS sample, and used Mass Spectroscopy; also, the time of their analysis was six minutes. In contrast, in our work, we detected amino acids using a fluorescence detector; furthermore, we avoided the presence of the interfering compounds due to the use of two chemical compounds: OPA and MPA reagents that only react with the amino group in the structure of the amino acids. Also, in comparison to Haghighi and other's research, the chromatograms we obtained were characterized by much smoother baseline (also for the standard), which indicates stable conditions in the HPLC unit. Also, the peaks obtained by the other group are relatively wide and with tails whereas in our results, the peaks are much sharper and the tails are absent. Tcherkas \& Denisenko (Tcherkas \& Denisenko, 2001) reported a method to measure two biologically
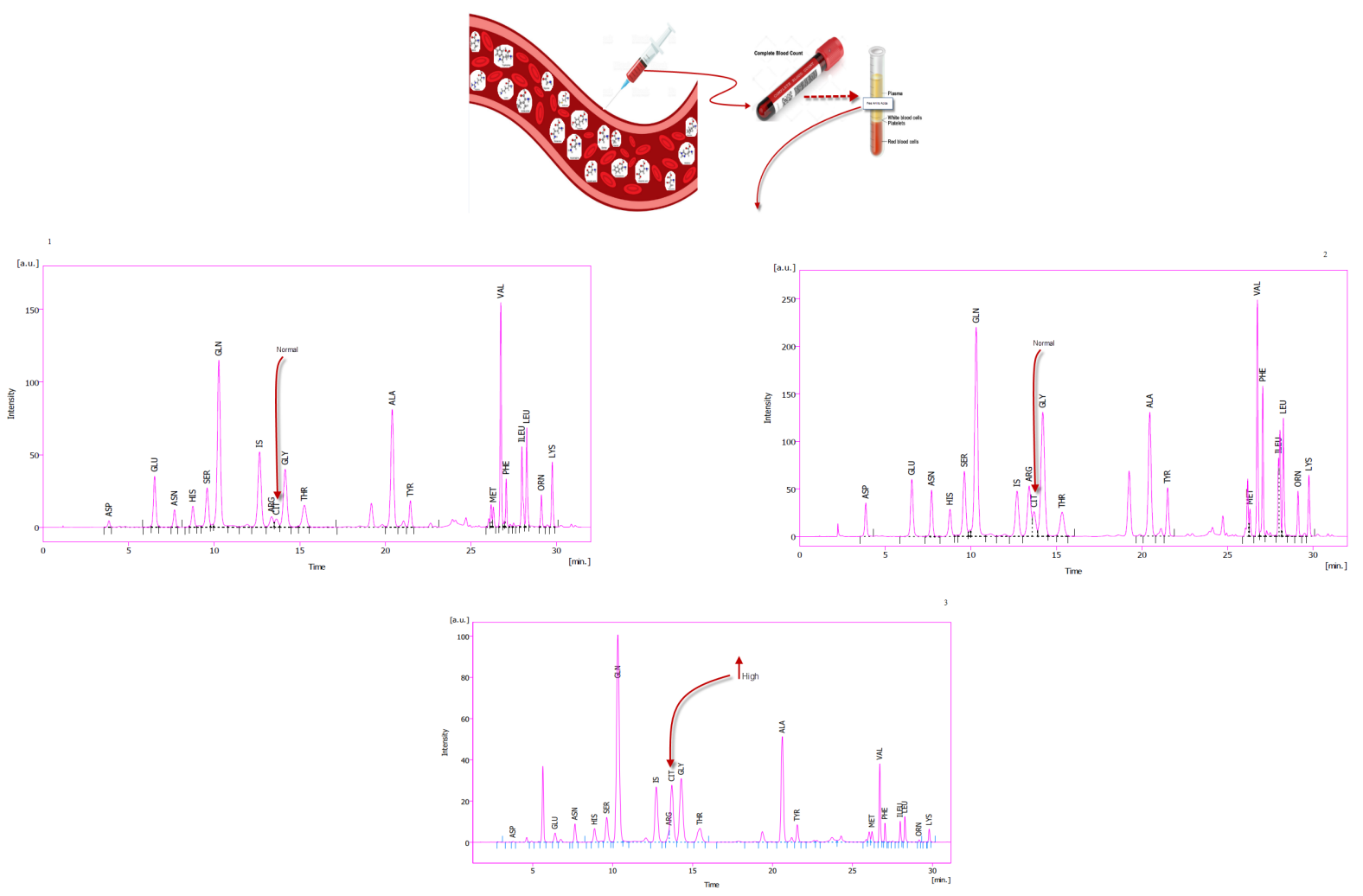

Figure 4. The HPLC procedure.

The plasma amino acids (free amino acids) in samples taken from the children. Plasma amino acids used for the HPLC analysis. Graphs one and two are for healthy infants. Graph three is for infants suffering from the disorder (argininosuccinic aciduria disorder). 
important thiol-compounds: homocysteine and cysteine as well as amino acids: aspartate and glutamate. Amino acids were derived using $\mathrm{O}$-phthalaldehyde and 2-mercaptoethanol (for alkylation of free sulfhydryl group with iodoacetic acid) and measured by RP-HPLC. The analysis was performed using O-phthalaldehyde, 2-mercaptoethanol, for $30 \mathrm{~min}$, using Sheri Serb ODS-2 column, isocratic method, phosphate buffer ( $\mathrm{pH}$ 7.0) 0.002 $\mathrm{M} \mathrm{Na}_{2}$ EDTA, (0.04 $\mathrm{M}$ sodium and $17 \%$ methanol) and a fluorescence detector (excitation $340 \mathrm{~nm}$, emission 450 $\mathrm{nm})$. The results of Tcherkas \& Denisenko's research were acceptable because the two thiol-compounds were identified, but as their derivatives are usually unstable, the measured concentration may not be accurate; therefore, we omitted the analysis of these compounds (L. Phenylalanine and tyrosine level was measured in the plasma and DBS samples using HPLC-FD by Roman Kand'ar \& Pavla Zakova (Kand'ár \& Žáková, 2009). The researchers used a mixture of methanol and deionized water $(5: 95 \mathrm{v} / \mathrm{v})$ as the mobile phase; also, they used a reverse-phase column (Lichrocart 125-4, Purospher RP-18e $5 \mu \mathrm{m})$, LC-10ADVP pump, SIL-Auto sampler, CTO-10ASVP, RF-10AXL fluorescence detector, and SLC-10AVP system controller. Also, the flow rate was $0.5 \mathrm{~mL} / \mathrm{min}$, the emission and excitation wavelengths were $282 \mathrm{~nm}$ and $260 \mathrm{~nm}$, respectively, and they used the isocratic method. In the protocol, the sample was prepared by incubation for ten minutes at $4^{\circ} \mathrm{C}$; then, centrifuged to remove the precipitate; the supernatant was filtered with a $0.2 \mu \mathrm{m}$ nylon filter and finally transferred to a $10 \mathrm{~mL}$ vial for the subsequent analysis. In comparison, in our work there was no incubation during the sample preparation, therefore the sample pretreatment and preparation was easier and faster. Also, after the addition of the internal standard and centrifugation, there was no need to filter the clear supernatant. Aromatic amino acids were measured by Ying Li and others (Li et al., 2011) with HPLC-FD. First, the samples were deproteinated and precipitated with perchloric acid; then, they were injected into a C18 column $(4.6 \mathrm{~mm}, 25 \mathrm{~mm}$, i.d. $5 \mu \mathrm{m})$. The mobile phase consisted of $10 \%$ acetonitrile in water $(\mathrm{v} / \mathrm{v})$, and the flow rate was $1 \mathrm{~mL} / \mathrm{min}$. Tyrosine, phenylalanine, and tryptophan concentration was evaluated in less than 10 minutes using $200-450 \mathrm{~nm}$ excitation wavelength, 250-500 $\mathrm{nm}$ emission; also, the baseline was acceptable, but no internal standard was used in this experiment. In contrast, in our work, we added an internal standard and measured the concentration of twenty amino acids in 32 minutes. Xi-ming Mo and others (Mo et al., 2013) performed the determination of phenylalanine and tyrosine concentration in peripheral capillary blood by HPLC-UV using Hypersil C8 column, $5 \%$ acetonitrile $(\mathrm{v} / \mathrm{v})$ as mobile phase, flow rate of $1.5 \mathrm{~mL} / \mathrm{min}$, and $210 \mathrm{~nm}$ wavelength; however, their chromatogram peaks were not very sharp and had a bit of tail.

In conclusion, firstly, neonates should be screened at birth for amino acid metabolic disorders which is routinely performed in developed countries, but not in developing countries such as Iran; therefore, the determination of the amino acid profile must be performed by the Ministry of Health of Iran. Secondly, the symptoms of these diseases are very similar to some common neonatal diseases, so the Iranian medical community must consider the diagnosis and treatment. Also, Iran has higher family marriages percentage compared to the average values in the world; therefore, the government should educate and encourage the people to perform genetic tests in order to prevent these diseases as they undoubtedly are a tremendous problem to families, communities, and health service.

Finally, we developed and described a very accurate protocol to acquire the amino acid profile and diagnose argininosuccinic aciduria.

\section{REFERENCES}

Biase De, Liu A, Yuzyuk T, Longo N, Pasquali M (2015) Quantitative amino acid analysis by liquid chromatography-tandem mass spectrometry: Implications for the diagnosis of Argininosuccinic Aciduria. Clin Chim Acta 10: 73-74. https://doi.org/10.1016/j. cca.2015.01.008

Chin Chen B, Hock Ngu L, Yunus Zabadeh MD (2010) Argininosuccinic aciduria: clinical and biochemical phenotype findings in Malaysian children. Malays J Pathol 32: 87-95

Erez A (2013) Argininosuccinic aciduria: from a monogenic to a complex disorder. Genet Med 15: 251-257. https://doi.org/10.1038/ gim.2012.166

Feuchtbaum L, Carter J, Dowray S, Currier R, Lorey F (2012) Birth prevalence of disorders detectable through newborn screening by race/ethnicity. Genet Med 14: 937-945. https://doi.org/10.1038/ gim.2012.76. Epub 2012 Jul 5

Haghighi F, Talebpour Z, Amini V, Ahmadzadeh A, Farhadpour M (2015) A fast high performance liquid chromatographic (HPLC) analysis of amino acid phenylketonuria disorder in dried blood spots and serum samples, employing C18 monolithic silica columns and photo diode array detection. Anal Methods 7: 7560-7567

Kand'ár R, Záková P (2009) Determination of phenylalanine and tyrosine in plasma and dried blood samples using HPLC with fluorescence detection. I Chromatogr B Analyt Technol Biomed Life Sci 877: 3926-3929. https://doi.org/10.1016/j.jchromb.2009.09.045

Kim D, Min Ko J, Kim Y-M, Seo GH, Kim G-H, Lee BH, Yoo H-W (2018) Low prevalence of Argininosuccinate lyase deficiency among inherited urea cycle disorders in Korea. J Hum Genet 63: 911-917. https://doi.org/10.1038/s10038-018-0467-2

Levin B, Mackay HMM, Oberholzer VG (1961) Argininosuccinic aciduria: An inborn error of amino acid metabolism. Arch Dis Child 36: 622-632. https://doi.org/10.1136/adc.36.190.622

Li Y, Tang A-G, Mu S (2011) HPLC-FLD determination of serum aromatic amino acids: application in chronic kidney disease patients. Clin Chim Acta 412: 1032-1035. https://doi.org/10.1016/j. cca.2011.02.015

Martin RJ, Fanaroff AA, Walsh MC (2006) Fanaroff and Martin's neonatal-perinatal medicine diseases of the fetus and infant. Arch Dis Child Fetal Neonatal. 8th edn, Vol I and II, 91: F468

Mo X-M, Li Y, Tang A-G, Ren Y-P (2013) Simultaneous determination of phenylalanine and tyrosine in peripheral capillary blood by HPLC with ultraviolet detection. Clin Biochem 46: 1074-1078. https://doi.org/10.1016/j.clinbiochem.2013.05.047

Nagamani S, Erez A, Lee B (2012) Response to Srilatha et al. Genet Med 14: 628. https://doi.org/10.1038/gim.2012.49

Nelson DL, Cox MM, Lehninger AL (2017) Lebninger Principles of Biochemistry. 7 th edn. W.H. Freeman, New York

Sharer JD, Biase ID, Matern D, Young S, Bennett MJ, Tolun AA (2018) Laboratory analysis of amino acids, 2018 revision: A technical standard of the American College of Medical Genetics and Genomics (ACMG). Genet Med 20: 1499-1507. https://doi. org/10.1038/s41436018-0328-6

Tcherkas YV, Denisenko AD (2001) Simultaneous determination of several amino acids, including homocysteine, cysteine and glutamic acid, in human plasma by isocratic reversed-phase high-performance liquid chromatography with fluorimetric detection. J Chromatogr A 913: 309-313. https://doi.org/10.1016/s0021-9673(00)01201-2

U.S. National Library of Medicine. https://www.nlm.nih.gov. 8600 Rockville Pike Bethesda, MD 20894 